\title{
Conceptual design of a portable robot inspiring elderly people to walk
}

\begin{abstract}
In this paper, we propose a portable robot "Tech Tech" that inspires elderly people to walk. Walk is preferable to maintain their healthy condition both physically and mentally. The human-robot interaction is important for increasing their motivation. Tech Tech talks to elderly people based on the data from various sensors: Tech Tech can judge weather and indoor-outdoor environments. It can also count steps. We took a stochastic approach in order to determine the actions of the robot. The robot could produce preferable impressions such as "interesting," "friendly" and "satisfactory." Experimental results demonstrate that the elderly people prefer long intervals in conversations and affirmative responses.
\end{abstract}

Keywords: Portable robot; Rehabilitation; Elderly people; Walk; Human-robot interaction; Bayes' theorem
Volume 3 Issue 3 - 2017

\author{
Yoshikazu Mori,Yuri Kumahara \\ Department of Intelligent Systems Engineering, Ibaraki \\ University, Japan
}

Correspondence: Yoshikazu Mori, Department of Intelligent Systems Engineering, Ibaraki University, Japan,

Email yoshikazu.mori.zen@vc.ibaraki.ac.jp

Received: September 25, 2017 | Published: October 16, 2017

\section{Introduction}

In recent Japan, elderly people have increased, even though the total population has decreased. The percentage of the elderly aged 65 and over rose to $26.7 \%$ in $2015,{ }^{1}$ and $18.6 \%$ of them live alone. ${ }^{2}$ The average time staying at home is approximately $20 \mathrm{hr}^{3}$ Social withdrawal of elderly people, which might lead to dementia, has become increasingly common. Recently various companies have developed communication robots for elderly people. Some robots are actually used today in nursing homes. However, such robots are not intended for use outdoors. These robots do not give elderly people sufficient motivation to go outdoors, even if they become good conversation partners. Their size and weight are important factors for walk together. Moreover the way of taking them for a walk is also important. We have developed a portable robot inspiring elderly people to walk for the purpose of helping them to maintain their healthy condition.

\section{Conceptual design}

Walking supports healthy lifestyles of elderly people. Many people take walks together with dogs; however, the care of a pet becomes a burden for elderly people. We designed a robot that has the following functions:

a. The robot can judge the weather conditions.

b. The robot can judge indoor/outdoor environments to ascertain whether the user has been outside or not.

c. The robot can communicate with the user.

d. The robot can count the steps.

We have developed the robot "Tech Tech" as shown in Figure 1. The size is $26(\mathrm{~L}) 13(\mathrm{~W}) 10(\mathrm{H}) \mathrm{cm}$, and the weight is $220 \mathrm{~g}$. A microcomputer (Coron; STM32, $72 \mathrm{MHz}$ ) and sensors are installed in a stuffed-toy-shaped pen case. A rain sensor system and CdS sense the weather, and the results are sent to PC using another microcomputer (SH7125, 50MHz) and XBee. An ultraviolet sensor senses the indoor/ outdoor environments, and an acceleration sensor is used for counting steps. For the conversation with the user, a microphone and a speaker are installed. The robot can move the tail to appeal its feeling and recognize the image of environment using a camera.

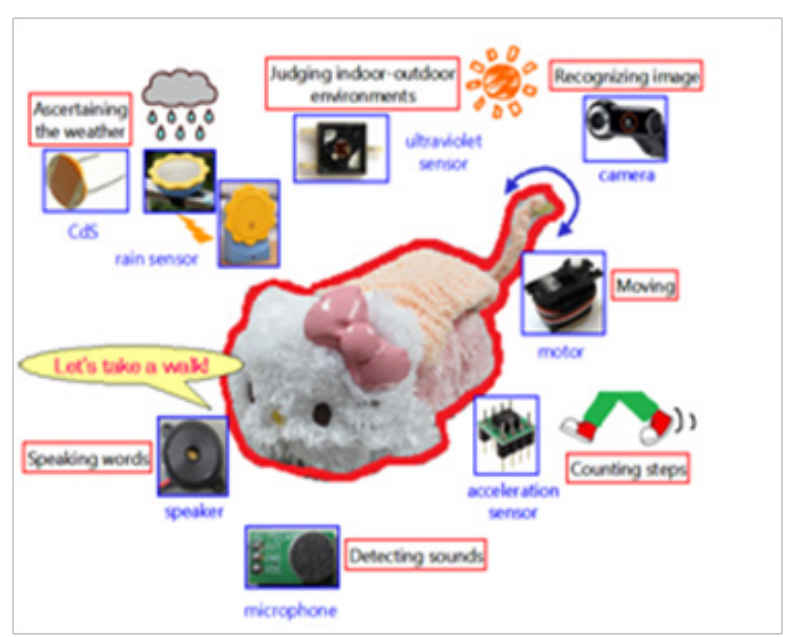

Figure I Hardware of "Tech Tech".

\section{Experiments}

We took a stochastic approach to determine the action of the robot. The procedure is the following:

a. The robot performs one of the following actions

I. Utter the line: Good weather isn't it. Let's take a walk!

II. Move the tail,

III. Perform (a) and (b) at the same time,

IV. Do nothing. After the robot

b. The robot performs 12 actions in all from the friendly above four patterns three times each randomly.

c. The relationship between the action of the robot and the response of the subject is calculated using Bayes' theorem as the following: 


$$
P(A \mid B)=\frac{P(A \cap B)}{P(B)}
$$

Where A is the response of walk, and B is the action of the robot Based on the results, action comfortable selection probabilities of the robot are determined: High probability actions are chosen more frequently. For example, if the times of walk for each action from (a) to (d) are $1,2,3$. and 0 , then the probabilities are set at $20 \%, 30 \%$, $45 \%$ and $5 \%$, respectively hateful Students.

Ten subjects were men and women: five subjects were in their $60 \mathrm{~s}$ to $70 \mathrm{~s}$, and five subjects, who were students in their 20s. They interacted with the robot five times. Moreover, they interacted with the robot whose actions were produced at random from the above four actions five times for comparison. The actions based on the stochastic approach induced the elderly people to walk 3.81 .30 times in average, while the number of the times of walk induced by the random actions was 2.20 .84 . The result of the impression is shown in Figure 2. The actions based on the stochastic approach were more "interesting," "friendly" and "satisfactory" than random actions. Next, we discuss the intervals and the responses in conversations. Ten subjects were men and women: five subjects were in their 60 s to 80 s, and five subjects, who were students in their 20s. Regarding the intervals in conversations, we tried three types: $0.3 \mathrm{~s}, 0.8 \mathrm{~s}$ and $1.3 \mathrm{~s}$. The $0.8 \mathrm{~s}$ interval $^{1}$ was based on the preliminary experimental results from students; they felt this interval was moderate. Subjects talked about self-introduction to the robot with different intervals. The result is shown in Figure 3. Elderly people tended to have a better impression for long intervals. Considering the responses in conversations, the robot responded in affirmative form or in question form when the subject talked about a certain topic with the robot. We confirmed that affirmative responses produced preferable impressions such as "satisfactory," "natural" and "human-like" for elderly people. In the long run, however, it will be necessary to use an appropriate combination of both of the forms.

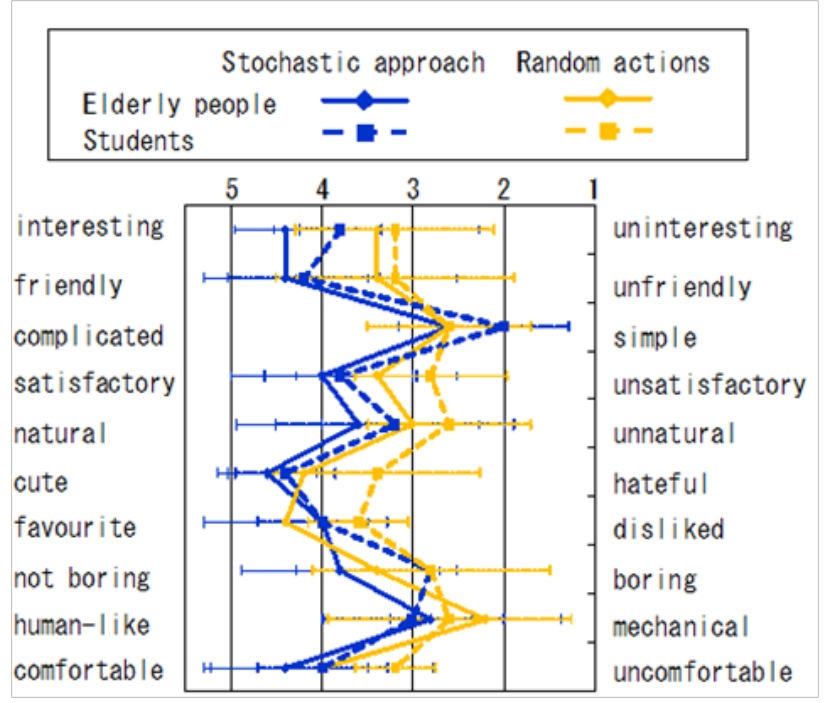

Figure 2 Impressions of the actions of "Tech Tech".

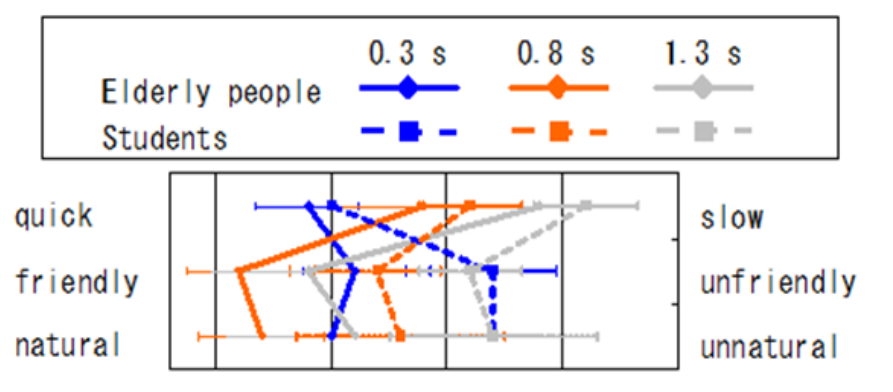

Figure 3 Impressions for various intervals in conversation.

\section{Conclusion}

We proposed a portable robot "Tech Tech" that walks with elderly people. Tech Tech talks to elderly people based on the data obtained from various sensors. We discussed effective method for inspiring elderly people to walk using Tech Tech. In the future we plan to develop strategy considering the sensitivity of older people.

\section{Acknowledgments}

No financial interest exists.

\section{Conflict of interest}

No conflict of interest exists.

\section{References}

1. Cabinet Office, Government of Japan. The Aging Society: Current Situation and Implementation Measures FY 2015. Annual Report on the Aging Society. 2015.

2. Ministry of Health Labour and Welfare. Summary Report of Comprehensive Survey of Living Conditions, Ministry of Health, Labour and Welfare. 2016.

3. Statistics Bureau. Survey on Time Use and Leisure Activities. 2011. 